\title{
The Solubility of Man-Made Mineral Fibers in the Lung in Contrast to Natural Fibers
}

\author{
D.M. Bernstein \\ Battelle, Geneva Research Centres, 7 route de Drize, 1227 Carouge-Geneva, Switzerland
}

R.T. Drew and M. Kuschner

School of Medicine, State University of New York at Stony Brook, Stony Brook, N.Y. 11794, USA

When inhaled, natural mineral fibers such as asbestos can produce pulmonary interstitial fibrosis and mesothelioma, and can contribute to the induction of lung cancer [1]. This is true in humans and in experimental animals. Animal studies with the man-made mineral fiber (MMMF), fiberglass, have shown some association with fibrosis and with the production of mesothelioma under the highly artificial method of pleural implantation. However, in contrast to experience with natural fibers, there has, as yet, been no clinical or epidemiological evidence that associates fiberglass with chronic lung disease.

The particular response to fibers is thought to be a product of fiber size [2] (length and diameter) and of their physico-chemical composition. The most carcinogenic forms of asbestos are largely of submicron diameter, with lengths greater than 10 to 20 micrometers. The small diameter allows substantial deposition in the alveolar region of the lung while the length hampers the ability of the macrophages to completely engulf and therefore clear the fibers. In addition, some studies indicate that the small diameters facilitate penetration of the fibers into the interstitial region of the lung, one of the primary sites of disease.

A series of studies has been performed with the support of the Thermal Insulation Manufacturers' Association (U.S.) to examine the deposition, translocation, as well as the biological and physical fate, of glass fibers and of crocidolite asbestos $[3,4]$. Fischer 344 rats were exposed by intratracheal instillation to glass fibers $1.5 \mu \mathrm{m}$ in diameter and of two different lengths, $5 \mu \mathrm{m}$ (short) and $50 \mu \mathrm{m}$ (long).

Following a single intratracheal instillation of $20 \mathrm{mg}$ the clearance curves of the long and short fibers are similar. The long-term clearance half-times were found to be 200 days and 140 days for the long and short fibers respectively. The biological response to the long fibers was the formation of foreign body granulomata that appear to be related to the large quantity of fibers and the method of administration. A much less severe response was seen in the animals exposed to the short fibers.

Scanning electron micrographs of the alveolar spaces demonstrate that the short fibers are found primarily within pulmonary macrophages. In contrast, long fibers are often seen in dense cellular masses. When long fibers were free in the lung, they could not be totally engulfed by macrophages and were often seen with two or three macrophages attempting to phagocytize a single fiber.

The dissolution of fibers in the lung was determined from the scanning electron micrographs by measuring the reduction in size of the fibers.

The results indicate that the long fibers stimulate an additional response in the lung which results in a significantly greater rate of dissolution. Short fibers were reduced in volume by $25 \%$ over 18 months while the long fibers were reduced in volume by nearly $50 \%$. The results suggest that this increased dissolution is associated with the macrophages' inability to completely phagocytize the long fibers. Those fibers that were observed partially phagocytized by macrophages were reduced in volume by almost $90 \%$.

This is in contrast to chrysotile asbestos in which the fiber remains similar to the original material but the surface and chemical properties are changed. Up to $35 \%$ of the structural magnesium has been shown to dissolve in one month in rats exposed by intraperitoneal inoculation [5]. It is also proposed that amphibole asbestos behaves in a similar manner with the metal ions being dissolved leaving a silicate skeleton.

The chemical composition and shape appear to be important determinants of the durability of the fiber. The substantial dissolution rate of glass fibers in the lung, especially when associated with macrophages may significantly lessen any long-term effects.

\section{REFERENCES}

Stanton MF and Wrench C. Mechanisms of mesothelioma induction with asbestos and fibrous glass. J. Nat, Can. Inst. 1972; 48: 797-821.

Wright $G W$ and Kuschner $M$. The influence of varying lengths of glass and asbestos fibers on tissue response in guinea pigs. In: Walton WH ed. Inhaled Particles IV. England: Pergamon Press, 1970 .

Bernstein DM et al. Experimental approaches for exposure to sized glass fibers. Env. Health Perspec. $1980 ; 34: 45-57$.

Bernstein DM et al. Pathogenicity of MMMF and the contrasts with natural fibers. In: Biological Effects of Man-Made Mineral Fibers, Proceedings of a WHO/ IARC Conference. ISBN 92890 10266, WHO, Geneva, 1984.

Morgan A et al. Studies of the solubility of constituents of chrysotile asbestos in vivo using radioactive tracer techniques. Environ. Res. 1981; 4: $558-570$.

Crawford $D$ and Miller $K$. Structure and structural changes in crocidolite asbestos associated with biological systems. Micron 1981; 12: 25-28. 